\title{
Influence of Professional Oral Hygiene Procedures on Plasma Glucose Levels of systemically Healthy Individuals: A Short-term Study
}

\author{
${ }^{1}$ Rithesh Kulal, ${ }^{2}$ Shashwati Paul, ${ }^{3}$ Savita Sambhashivaiah, ${ }^{4}$ Shilpa Shivanand
}

\begin{abstract}
Aims: The treatment of gingival inflammation is associated with improved glycemic control in diabetic patients. The aim of this study was to test the short-term effects of professional oral hygiene procedures on plasma glucose levels in systemically healthy individuals with periodontal diseases.
\end{abstract}

Materials and methods: Forty-five systemically healthy individuals visiting the RajaRajeswari Dental College \& Hospital were randomly allocated into three groups, group I (gingivitis), group II (periodontitis), and group III (healthy control). The subjects of groups I and II were treated by scaling and root planning whereas the healthy control group did not receive any treatment. The plasma glucose levels were estimated at baseline for all the three groups. Plasma glucose levels were again assessed immediately following the oral hygiene procedures and at 1 week recall visit for groups I and II.

Results: All 45 patients completed the study. There were no dropouts. There was no statistically significant change in random blood sugar (RBS) level in group I (gingivitis) from baseline to postoperative whereas there was a statistically significant reduction in the mean RBS levels from postoperative to 1 week. In group II (periodontitis), there was a significant reduction in the mean values of RBS from baseline to postoperative and baseline to 1 week.

Conclusion: This short-term study showed that professional oral hygiene procedures are effective in improving the glycemic status of systemically healthy patients with periodontal diseases.

Keywords: Gingivitis, Glucose, Periodontitis.

How to cite this article: Kulal R, Paul S, Sambhashivaiah S, Shivanand S. Influence of Professional Oral Hygiene Procedures on Plasma Glucose Levels of systemically Healthy Individuals: A Short-term Study. J Health Sci Res 2015;6(2):29-32.

Source of support: Nil

Conflict of interest: None

\footnotetext{
${ }^{1}$ Reader, ${ }^{2,4}$ Postgraduate Student, ${ }^{3}$ Professor and Head

${ }^{1-4}$ Department of Periodontology, RajaRajeswari Dental College \& Hospital, Bengaluru, Karnataka, India

Corresponding Author: Shashwati Paul, Postgraduate Student, Department of Periodontology, RajaRajeswari Dental College \& Hospital, Bengaluru, Karnataka, India, Phone: +918951502864, e-mail: hishashwati@gmail.com
}

\section{INTRODUCTION}

Gingivitis and periodontitis are two most common diseases of oral cavity. Gingivitis that is associated with plaque is the most common form of gingival disease. Periodontitis is an inflammatory disease of the supporting tissues of the teeth, caused by specific microorganisms and resulting in progressive destruction of periodontal ligament and alveolar bone with pocket formation, recession, or both. Gingivitis is able to evoke a specific host response at a periodontal site, but the type of systemic host response in patient with gingival inflammation is still not clear. Experimental gingivitis was associated with endotoxemia and hyperactivity of circulatory neutrophils but not with systemic levels of cytokines and acute phase proteins. ${ }^{1}$ Gingival inflammation and infection of periodontium lead to worsening of glycemia control in patients with diabetes. ${ }^{2}$ Some evidence suggests the resolution of gingival inflammation could improve glycemic control in type 1 diabetic patients, checked by blood glucose and glycosylated hemoglobin levels. ${ }^{3-5} \mathrm{Few}$ studies have been done to investigate whether the treatment of gingivitis and periodontitis is associated with changes in blood glucose levels in systemically healthy individuals. The aim of this study was to assess the shortterm effects of professional oral hygiene procedures on plasma glucose level of systemically healthy individuals with periodontal diseases.

In this study, we evaluated plasma glucose levels in systemically healthy individuals with gingivitis and periodontitis. We compared the plasma glucose level in patients with gingivitis and periodontitis with the healthy controls and also compared the short-term effects of oral hygiene procedures on plasma glucose levels in subjects with gingivitis and periodontitis.

\section{MATERIALS AND METHODS}

A total of 45 patients, both male and female in the age group of 18 to 60 years visiting RajaRajeswari Dental College \& Hospital, Bengaluru, were recruited for the study. The subjects were randomly divided into three groups:

Group I: Fifteen subjects with untreated chronic generalized gingivitis. 
Group II: Fifteen subjects with untreated chronic periodontitis.

Group III: Fifteen subjects with healthy periodontium.

The inclusion criteria for group I (chronic generalized gingivitis) was untreated chronic generalized gingivitis with presence of clinical signs of inflammation with no attachment loss. The inclusion criteria for group II (chronic periodontitis) was untreated chronic periodontitis with a probing depth $>5 \mathrm{~mm}$, clinical attachment loss $\geq 3 \mathrm{~mm}$, and radiographic evidence of alveolar bone loss on at least two teeth per quadrant, excluding the third molars.

Patients with history of systemic diseases which affects the periodontium, smokers and alcoholic patients, and patients on immunosuppressive agents or antibiotics taken within 6 months were excluded from the study. Pregnant and lactating mothers and patients who have undergone periodontal treatment within a period of 1 year were also not included in the study.

All the patients underwent a full-mouth periodontal probing and charting and was screened for their suitability for the study. Clinical parameters which were recorded during screening included plaque index (PI), gingival index (GI), and probing pocket depth (PPD), which was measured using graduated Williams periodontal probe from the crest of gingival margin to base of the pocket.

The subjects of groups I and II were treated by scaling and root planning, whereas the healthy control group did not receive any treatment. The plasma glucose levels were estimated at baseline for all the three groups. Plasma glucose levels were again assessed immediately following the oral hygiene procedures and at 1 week recall visit for groups I and II.

\section{Plasma Glucose Level Estimation}

Plasma glucose levels were assessed using a commercial glucometer (Accu-check) by piercing the skin on the finger to draw a blood drop, then applying it to the chemically active disposable "test-strip" of the glucometer. Blood glucose levels were assessed at baseline, immediately after the procedure, and 1 week after oral hygiene procedures.

\section{Statistical Analysis}

The results were statistically analyzed using Kruskal-Wallis analysis of variance and Wilcoxon matched pairs test.

\section{RESULTS}

All the 45 patients completed the study. Graph 1 shows the comparison of blood glucose levels of the three groups at baseline. The mean random blood sugar (RBS) levels of

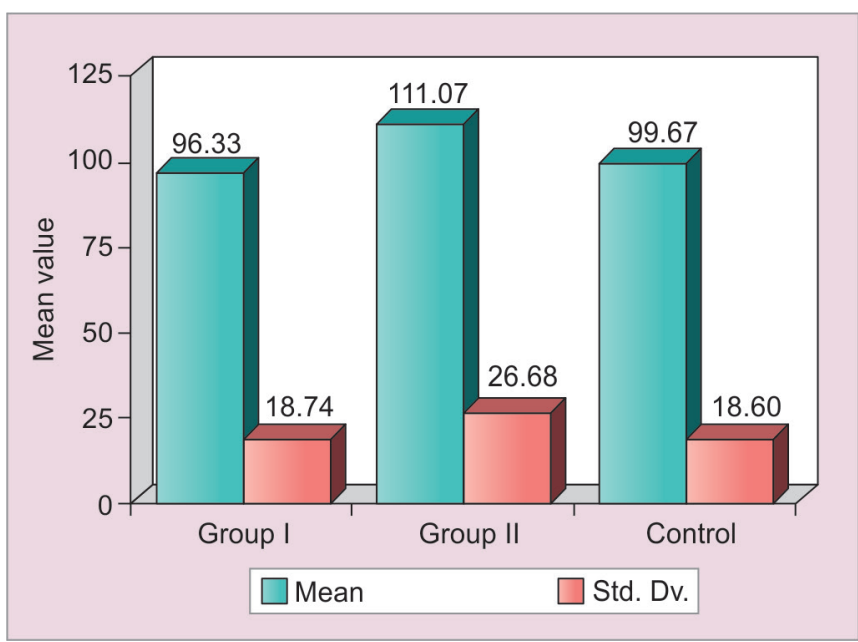

Graph 1: Comparison of three groups (I, II, control) with respect to baseline RBS levels

group I (gingivitis) was $96.33 \mathrm{mg} / \mathrm{dL}$. In the periodontitis group, the mean RBS level was $111.07 \mathrm{mg} / \mathrm{dL}$, also within the normal laboratory values, but was higher compared to the healthy control group $(99.67 \mathrm{mg} / \mathrm{dL})$.

Graph 2 shows the comparison of blood glucose levels in group I at baseline, postoperative, and at 1 week recall visit. There was no statistically significant change in RBS level in group I (gingivitis) from baseline to postoperative, whereas there was a statistically significant reduction in the mean RBS levels from postoperative to 1 week.

Graph 3 shows the comparison of blood glucose levels in group II at baseline, postoperative, and at 1 week interval. In group II (periodontitis), there was a significant reduction in the mean values of RBS from baseline to postoperative and baseline to 1 week.

\section{DISCUSSION}

In this study, the mean RBS levels of group I (gingivitis) was $96.33 \mathrm{mg} / \mathrm{dL}$. In the periodontitis group, the mean

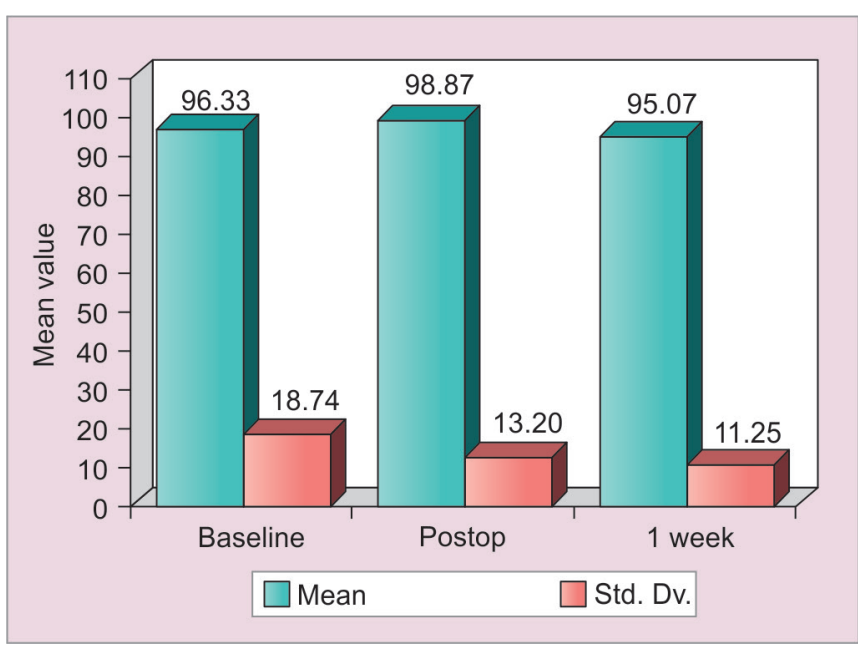

Graph 2: Comparison of baseline, postoperative, and 1 week with respect to RBS levels in group I 


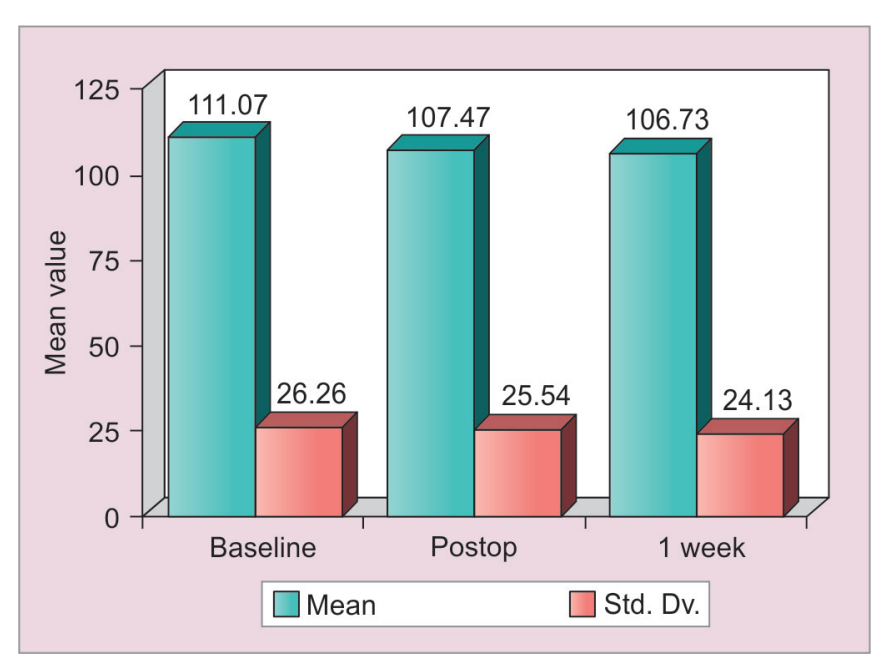

Graph 3: Comparison of baseline, postoperative, and 1 week with respect to RBS levels in group II

RBS level was $111.07 \mathrm{mg} / \mathrm{dL}$, also within the normal laboratory values, but was higher compared to the healthy control group $(99.67 \mathrm{mg} / \mathrm{dL})$. There was no statistically significant change in RBS level in group I (gingivitis) from baseline to postoperative, whereas there was a statistically significant reduction in the mean RBS levels from postoperative to 1 week. In group II (periodontitis), there was a significant reduction in the mean values of RBS from baseline to postoperative and baseline to 1 week. The results of this study show that, following periodontal therapy, there is a statistically significant improvement in glycemic control.

The association between periodontitis and diabetes has been explored by many researchers over the years. ${ }^{6-9}$ In this study, the periodontitis group was associated with a higher mean RBS levels even in systemically healthy subjects. Evidence suggests that the local inflammatory or infectious factors might trigger a systemic host response, therefore predisposing subjects with periodontitis to an increased risk of systemic diseases. ${ }^{10}$

Certain studies have been conducted on diabetic patients to evaluate the effect of nonsurgical therapy on the glycemic levels. In 2001, a study done by Stewart et $\mathrm{al}^{4}$ reported a decrease in the blood glucose levels following nonsurgical therapy of periodontitis in type 2 diabetes mellitus patients.

Singh et a ${ }^{11}$ studied the effect of periodontal therapy on the improvement of glycemic control in patients with type 2 diabetes mellitus. The results obtained appear to demonstrate a strong, statistically significant, association between clinical improvement in the periodontal condition and improved metabolic control of diabetes.

Some studies failed to show any significant improvement in blood glucose levels following nonsurgical periodontal therapy. Shaikh and Shirahatti ${ }^{12}$ conducted a pilot study to assess the effects of scaling and root planning on blood glucose levels in type 2 diabetes patients and found no significant change in the fasting and postprandial blood glucose levels in patients treated with scaling and root planning.

Few studies have been done to assess the effect of nonsurgical periodontal therapy like scaling and root planning on blood glucose levels in systemically healthy patients. Nieri et $\mathrm{al}^{13}$ conducted a study in 2014 to test the short-term effects of professional oral hygiene procedures on plasma glucose levels in systemically healthy individuals. The study concluded that professional oral hygiene procedures are not able to influence plasma glucose levels in individuals with gingivitis, but otherwise systemically healthy.

However, the results of this study suggest that periodontitis can influence the glycemic status in systemically healthy individuals, and successful management of periodontal infection will lead to a reduction of the local symptoms of the disease and better control of glucose metabolism.

\section{CONCLUSION}

Within the limits of this study, we concluded that periodontitis influences the glycemic status in systemically healthy individuals. However, gingivitis does not show significant effect on the glycemic levels. Professional oral hygiene procedures are effective in improving the periodontal status and also in improving the glycemic status of patients.

\section{REFERENCES}

1. Wahaidi VY, Kowolik MJ, Eckert GJ, Galli DM. Endotoxemia and the host systemic response during experimental gingivitis. J Clin Periodontol 2011 May;38(5):412-417.

2. Llambes F, Arias-Herrera S, Caffesse R. Relationship between diabetes and periodontal infection. World J Diabetes 2015; Jul 10;6(7):927-935.

3. Kiran M, Arpak N, Unsal E, Erdogan MF. The effect of improved periodontal health on metabolic control in type 2 diabetes mellitus. J Clin Periodontol 2005 Mar;32(3): 266-272.

4. Stewart JE, Wager KA, Friedlander AH, Zadeh HH. The effect of periodontal treatment on glycemic control in patients with type 2 diabetes mellitus. J Clin Periodontol 2001 Apr; 28(4):306-310.

5. Janket SJ, Wightman A, Baird AE, Van Dyke TE, Jones JA. Does periodontal treatment improve glycemic control in diabetic patients? A meta-analysis of intervention studies. J Dent Res 2005 Dec;84(12):1154-1159.

6. Bartolucci EG, Parkes RB. Accelerated periodontal breakdown in uncontrolled diabetes: pathogenesis and treatment. Oral Surg Oral Med Oral Pathol 1981 Oct;52(4):387-390.

7. Rylander H, Ramberg P, Blohme G, Lindhe J. Prevalence of periodontal disease in young diabetics. J Clin Periodontol 1987 Jan;14(1):38-43. 
8. Seppala B, Seppala M, Ainamo J. A longitudinal study on insulin-dependent diabetes mellitus and periodontal disease. J Clin Periodontol 1993 Mar;20(3):161-165.

9. Novaes AB Jr, Pereira A, Novaes AB. Manifestation of insulindependent diabetes mellitus in the periodontium of young Brazilian patients. J Periodontol 1991 Feb;62(2):116-122.

10. Li X, Kolltveit KM, Tronstad L, Olsen I. Systemic diseases caused by oral infection. Clin Microbial Rev 2000 Oct;13(4): 547-558.

11. Singh S, Kumar V, Kumar S, Subbappa A. The effect of periodontal therapy on the improvement of glycemic control in patients with type 2 diabetes mellitus: a randomized controlled clinical trial. Int J Diabetes Dev Ctries 2008 Apr-Jun; 28(2):38-44.

12. Shaikh UR, Shirahatti RV. Assessment of the effects of scaling and root planing on blood glucose levels in type II diabetes patients: a pilot study. J Int Clin Dent Res Organ 2010;2:20-23.

13. Nieri M, Pagliaro U, Giani M, Pennati V, Tonelli P, Cairo F. Professional oral hygiene procedures do not influence plasma glucose levels in systemically healthy individuals: a shortterm, randomised, controlled trial. Eur J Oral Implantol 2014 Spring;7(1):79-85. 